\title{
On lacunary statistical boundedness
}

\author{
Vinod K Bhardwaj ${ }^{1}$, Sandeep Gupta², Syed A Mohiuddine ${ }^{3}$ and Adem Kılıçman ${ }^{4,5 *}$
}

${ }^{\text {"Correspondence: }}$ akilic@upm.edu.my

${ }^{4}$ Department of Mathematics, Universiti Putra Malaysia, Serdang,

Selangor 43400, Malaysia

${ }^{5}$ Institute for Mathematical

Research, Universiti Putra Malaysia,

Serdang, Selangor 43400, Malaysia

Full list of author information is

available at the end of the article

\begin{abstract}
A new concept of lacunary statistical boundedness is introduced. It is shown that, for a given lacunary sequence $\theta=\left\{k_{r}\right\}$, a sequence $\left\{x_{k}\right\}$ is lacunary statistical bounded if and only if for 'almost all $k$ w.r.t. $\theta$ ', the values $x_{k}$ coincide with those of a bounded sequence. Apart from studying various algebraic properties and computing the Köthe-Toeplitz duals of the space $S_{\theta}(b)$ of all lacunary statistical bounded sequences, a decomposition theorem is also established. We characterize those $\theta$ for which $S_{\theta}(b)=S(b)$. Finally, we give a general description of inclusion between two arbitrary lacunary methods of statistical boundedness.

MSC: 40C05; 40A05; 46A45
\end{abstract}

Keywords: lacunary sequence; statistical boundedness; statistical convergence; Köthe-Toeplitz duals

\section{Introduction and background}

Statistical convergence is a generalization of the usual notion of convergence. The idea of statistical convergence was given in the first edition (published in Warsaw in 1935) of the monograph of Zygmund [1], who called it 'almost convergence.' Formally the concept of statistical convergence was introduced by Fast [2] in the year 1951 and later reintroduced by Schoenberg [3] in the year 1959. Statistical convergence also arises as an example of 'convergence in density' as introduced by Buck [4].

Although statistical convergence was introduced over nearly last 60 years, it has become an active area of research in recent years. Statistical convergence has been studied most recently by several authors [5-25].

The standard definition of ' $\left\{x_{k}\right\}$ is convergent to $L$ ' requires that the set $\left\{k \in \mathbb{N}:\left|x_{k}-L\right| \geq\right.$ $\varepsilon$ \} should be finite for every $\varepsilon>0$, where $\mathbb{N}$ is the set of natural numbers.

The number sequence $\left\{x_{k}\right\}$ is said to be statistically convergent to the number $L$ provided that the set $\left\{k \in \mathbb{N}:\left|x_{k}-L\right| \geq \varepsilon\right\}$, instead of being finite, has natural density 0 , where the natural density of a subset $K \subset \mathbb{N}$ (see [26], Chapter 11) is defined by

$$
\delta(K)=\lim _{n \rightarrow \infty} \frac{1}{n}|\{k \leq n: k \in K\}|,
$$

where $|\{k \leq n: k \in K\}|$ denotes the number of elements of $K$ not exceeding $n$. A set $K$ is said to be statistically dense [27] if $\delta(K)=1$. A subsequence of a sequence is said to be statistically dense if the set of all indices of its elements is statistically dense. Obviously, we have $\delta(K)=0$ provided that $K$ is a finite set of positive integers.

We shall be particularly concerned with those subsets of $\mathbb{N}$ which have natural density zero. To facilitate this, Fridy [10] introduced the following notation: if $x=\left\{x_{k}\right\}$ is a sequence

C2014 Bhardwaj et al.; licensee Springer. This is an Open Access article distributed under the terms of the Creative Commons Attribution License (http://creativecommons.org/licenses/by/2.0), which permits unrestricted use, distribution, and reproduction in any medium, provided the original work is properly cited. 
such that $x_{k}$ satisfies property $\mathrm{P}$ for all $k$ except a set of natural density zero, then we say that $x=\left\{x_{k}\right\}$ satisfies $\mathrm{P}$ for 'almost all $k$ ' and we abbreviate this by 'a.a.k'.

Using this notation, we have the following.

Definition 1.1 The number sequence $x=\left\{x_{k}\right\}$ is said to be statistically convergent to the number $L$ if for each $\varepsilon>0$,

$$
\delta\left(A_{\varepsilon}\right)=0, \quad \text { where } A_{\varepsilon}=\left\{k \in \mathbb{N}:\left|x_{k}-L\right| \geq \varepsilon\right\},
$$

i.e.,

$$
\lim _{n \rightarrow \infty} \frac{1}{n}\left|\left\{k \leq n:\left|x_{k}-L\right| \geq \varepsilon\right\}\right|=0,
$$

i.e.,

$$
\left|x_{k}-L\right|<\varepsilon \quad \text { a.a.k. }
$$

Following Freedman et al. [28], by a lacunary sequence $\theta=\left\{k_{r}\right\}_{r=0}^{\infty}$, where $k_{0}=0$, we shall mean an increasing sequence of non-negative integers with $k_{r}-k_{r-1} \rightarrow \infty$ as $r \rightarrow \infty$. The intervals determined by $\theta$ will be denoted by $I_{r}=\left(k_{r-1}, k_{r}\right]$, and we let $h_{r}=k_{r}-k_{r-1}$. Sums of the form $\sum_{i=k_{r-1}+1}^{k_{r}}\left|x_{i}\right|=\sum_{i \in I_{r}}\left|x_{i}\right|$ will be written for convenience as $\sum_{I_{r}}\left|x_{i}\right|$ and the ratio $\frac{k_{r}}{k_{r-1}}$ will be denoted by $q_{r}$.

There is a strong connection [28] between the space $\left|\sigma_{1}\right|$ of strongly Cesàro summable sequences:

$$
\left|\sigma_{1}\right|=\left\{x=\left\{x_{k}\right\}: \text { there exists } L \text { such that } \frac{1}{n} \sum_{k=1}^{n}\left|x_{k}-L\right| \rightarrow 0\right\}
$$

and the sequence space $N_{\theta}$, which is defined by

$$
N_{\theta}=\left\{x=\left\{x_{k}\right\}: \text { there exists } L \text { such that } \frac{1}{h_{r}} \sum_{I_{r}}\left|x_{k}-L\right| \rightarrow 0\right\} .
$$

Fridy and Orhan [29] introduced and studied a concept of convergence, called lacunary statistical convergence, that is related to statistical convergence in the same way as $N_{\theta}$ is related to $\left|\sigma_{1}\right|$.

Definition 1.2 Let $\theta$ be a lacunary sequence. The number sequence $x=\left\{x_{k}\right\}$ is lacunary statistical convergent or $S_{\theta}$-convergent to $L$ provided that for every $\varepsilon>0, \lim _{r \rightarrow \infty} \frac{1}{h_{r}} \mid\{k \in$ $\left.I_{r}:\left|x_{k}-L\right| \geq \varepsilon\right\} \mid=0$. In this case, we write $S_{\theta}-\lim x=L$ or $x_{k} \rightarrow L\left(S_{\theta}\right)$, and we define $S_{\theta}=\left\{x\right.$ : for some $\left.L, S_{\theta}-\lim x=L\right\}$.

Definition 1.3 For a lacunary sequence $\theta=\left\{k_{r}\right\}$, we define the lacunary density or $\theta$ density of $K \subset \mathbb{N}$ by

$$
\delta^{\theta}(K)=\lim _{r \rightarrow \infty} \frac{1}{h_{r}}\left|\left\{k \in I_{r}: k \in K\right\}\right| .
$$


Remark 1.4 For a non-negative regular matrix $A=\left(a_{n k}\right)$, following Freedman and Sember [30], Demirci [8] defined the concept of $A$-density for a set $K \subset \mathbb{N}$ as

$$
\delta_{A}(K)=\lim _{n \rightarrow \infty}\left\{A \chi_{K}\right\}_{n}
$$

where $\chi_{K}$ is the characteristic function of $K$.

For $A=C_{1}$, the Cesàro mean of order one, $\delta_{A}(K)$ reduces to $\delta(K)$, i.e., the natural density of the set $K$.

For a lacunary sequence $\theta=\left\{k_{r}\right\}$, if we take $A=\left(a_{n k}\right)$ such that

$$
a_{n k}= \begin{cases}\frac{1}{h_{n}}, & \text { if } k \in I_{n} ; \\ 0, & \text { otherwise, }\end{cases}
$$

then $\delta_{A}(K)$ reduces to $\delta^{\theta}(K)$, i.e., the lacunary density or $\theta$ density of $K$. Thus the lacunary density or $\theta$ density is a particular case of the $A$-density.

We now introduce the following notation.

For a given lacunary sequence $\theta=\left\{k_{r}\right\}$, if $x=\left\{x_{k}\right\}$ is a sequence such that $x_{k}$ satisfies property P for all $k$, except a set of $\theta$ density zero, then we say that $x=\left\{x_{k}\right\}$ satisfies P for 'almost all $\mathrm{k}$ with respect to $\theta$ ' and we abbreviate this by 'a.a.k w.r.t. $\theta$ '.

Using this notation, we have the following.

Definition 1.5 Let $\theta=\left\{k_{r}\right\}$ be a lacunary sequence. The number sequence $x=\left\{x_{k}\right\}$ is said to be lacunary statistical convergent or $S_{\theta}$-convergent to $L$ provided that for every $\varepsilon>0$,

$$
\left|x_{k}-L\right|<\varepsilon \quad \text { a.a.k w.r.t. } \theta \text {. }
$$

In 1997, Fridy and Orhan [31] introduced the concept of statistical boundedness as follows.

Definition 1.6 The number sequence $x=\left\{x_{k}\right\}$ is statistically bounded if there is a number $M>0$ such that $\delta\left(\left\{k:\left|x_{k}\right|>M\right\}\right)=0$, i.e.,

$$
\left|x_{k}\right| \leq M \quad \text { a.a.k. }
$$

We denote the set of all statistically bounded sequences by $S(b)$.

In the same year, i.e., 1997, Tripathy [32] proved a decomposition theorem for statistically bounded sequences and also established a necessary and sufficient condition for a sequence to be statistically bounded.

Quite recently, Bhardwaj and Gupta [33] have introduced and studied the concepts of statistical boundedness of order $\alpha, \lambda$-statistical boundedness and $\lambda$-statistical boundedness of order $\alpha$.

The main object of this paper is to introduce and study the new concept of lacunary statistical boundedness. 
Definition 1.7 Let $\theta=\left\{k_{r}\right\}$ be a lacunary sequence. The number sequence $x=\left\{x_{k}\right\}$ is said to be lacunary statistical bounded or $S_{\theta}$-bounded if there exists $M>0$ such that

$$
\lim _{r \rightarrow \infty} \frac{1}{h_{r}}\left|\left\{k \in I_{r}:\left|x_{k}\right|>M\right\}\right|=0,
$$

i.e.,

$$
\delta^{\theta}\left(\left\{k \in \mathbb{N}:\left|x_{k}\right|>M\right\}\right)=0,
$$

i.e.,

$$
\left|x_{k}\right| \leq M \quad \text { a.a.k w.r.t. } \theta \text {. }
$$

For a given lacunary sequence $\theta=\left\{k_{r}\right\}$, by $S_{\theta}(b)$ we denote the set of all $S_{\theta}$-bounded sequences. Obviously, $S_{\theta}(b)$ is a linear space with respect to co-ordinatewise addition and scalar multiplication.

In the next section we establish elementary relations among the concepts of boundedness, lacunary statistical boundedness and lacunary statistical convergence of sequences of numbers. It is shown that for a given lacunary sequence $\theta=\left\{k_{r}\right\}$, a sequence $\left\{x_{k}\right\}$ is lacunary statistical bounded if and only if for almost all $k$ w.r.t. $\theta$, the values $x_{k}$ coincide with those of a bounded sequence. Apart from studying various algebraic properties and computing the Köthe-Toeplitz duals of the sequence space $S_{\theta}(b)$, a decompositon theorem for lacunary statistical boundedness is also established. In Section 3, we characterize those $\theta$ for which $S_{\theta}(b)=S(b)$. It is also shown that precisely those subsequences of a lacunary statistical bounded sequence are lacunary statistical bounded which are lacunary statistical dense. In the last section, we consider the inclusion of $S_{\theta^{\prime}}(b)$ by $S_{\theta}(b)$ where $\theta^{\prime}$ is a lacunary refinement of $\theta$. Recall [28] that the lacunary sequence $\theta^{\prime}=\left\{k_{r}^{\prime}\right\}$ is called a lacunary refinement of the lacunary sequence $\theta=\left\{k_{r}\right\}$ if $\left\{k_{r}\right\} \subset\left\{k_{r}^{\prime}\right\}$. A general description of inclusion between two arbitrary lacunary methods of statistical boundedness is also given.

Before proceeding to establish the proposed results, we pause to collect some more definitions, which may be conveniently found in $[34,35]$.

A sequence space $X$ is called

(i) normal (or solid) if $y=\left\{y_{k}\right\} \in X$ whenever $\left|y_{k}\right| \leq\left|x_{k}\right|, k \geq 1$, for some $x=\left\{x_{k}\right\} \in X$,

(ii) monotone if it contains the canonical preimages of all its stepspaces,

(iii) sequence algebra if $x y=\left\{x_{k} y_{k}\right\} \in X$ whenever $x=\left\{x_{k}\right\}, y=\left\{y_{k}\right\} \in X$.

The idea of dual sequence spaces was introduced by Köthe and Toeplitz [36] whose main results concerned $\alpha$-duals; the $\alpha$-dual of sequence space $X$ being defined as

$$
X^{\alpha}=\left\{a=\left\{a_{k}\right\}: \sum_{k}\left|a_{k} x_{k}\right|<\infty \text { for all } x=\left\{x_{k}\right\} \in X\right\} .
$$

In the same paper [36], they also introduced another kind of dual, namely, the $\beta$-dual (see [37] also, where it is called the g-dual by Chillingworth) defined as

$$
X^{\beta}=\left\{a=\left\{a_{k}\right\}: \sum_{k} a_{k} x_{k} \text { converges for all } x=\left\{x_{k}\right\} \in X\right\} .
$$


Obviously $\phi \subset X^{\alpha} \subset X^{\beta}$, where $\phi$ is the well-known sequence space of finitely non-zero scalar sequences. Also if $X \subset Y$, then $Y^{\eta} \subset X^{\eta}$ for $\eta=\alpha$ or $\beta$. For any sequence space $X$, we denote $\left(X^{\delta}\right)^{\eta}$ by $X^{\delta \eta}$ where $\delta, \eta=\alpha$ or $\beta$. It is clear that $X \subset X^{\eta \eta}$ where $\eta=\alpha$ or $\beta$.

For a sequence space $X$, if $X=X^{\alpha \alpha}$ then $X$ is called a Köthe space or a perfect sequence space.

\section{Some inclusion relations and a decomposition theorem}

We begin by establishing elementary connections between boundedness, lacunary statistical boundedness and lacunary statistical convergence.

We state the following result without proof in view of the fact that the empty set has zero lacunary density for every lacunary sequence $\theta$.

Theorem 2.1 Every bounded sequence is lacunary statistical bounded, i.e., $\ell_{\infty} \subset S_{\theta}(b)$ for every lacunary sequence $\theta$.

Remark 2.2 The converse of the above theorem need not be true.

Example 2.3 Define $x=\left\{x_{i}\right\}$ as

$$
x_{i}= \begin{cases}k_{r-1}+1, & \text { if } i=k_{r-1}+1, r=1,2,3, \ldots \\ 0, & \text { otherwise }\end{cases}
$$

where $\theta=\left\{k_{r}\right\}$ is a lacunary sequence. Clearly $x=\left\{x_{i}\right\} \notin \ell_{\infty}$. But $\lim _{r \rightarrow \infty} \frac{1}{h_{r}} \mid\left\{k \in I_{r}:\left|x_{k}\right|>\right.$ $\left.\frac{1}{2}\right\} \mid=\lim _{r \rightarrow \infty} \frac{1}{h_{r}}=0$ and so $x=\left\{x_{i}\right\} \in S_{\theta}(b)$.

Remark 2.4 From Theorem 2.1 and above example, it is clear that lacunary statistical boundedness is a generalization of the usual concept of boundedness of sequences.

Theorem 2.5 Every lacunary statistical convergent sequence is lacunary statistical bounded, but not conversely.

Proof Let $x_{k} \rightarrow L\left(S_{\theta}\right)$. Then for each $\varepsilon>0$, we have $\lim _{r \rightarrow \infty} \frac{1}{h_{r}}\left|\left\{k \in I_{r}:\left|x_{k}-L\right| \geq \varepsilon\right\}\right|=0$. The result now follows from the fact that $\left\{k \in I_{r}:\left|x_{k}\right| \geq|L|+\varepsilon\right\} \subset\left\{k \in I_{r}:\left|x_{k}-L\right| \geq \varepsilon\right\}$.

It can easily be verified that lacunary statistical bounded sequence $\left\{(-1)^{k}\right\}$ is not lacunary statistical convergent.

Remark 2.6 There are sequences which are not lacunary statistical bounded for any lacunary sequence $\theta$. One example of such sequences is $x=\left\{x_{k}\right\}$ where $x_{k}=k$ for each $k \in \mathbb{N}$. Hence $S_{\theta}(b) \neq \omega$ where $\omega$ is the space of all scalar sequences.

Theorem 2.7 For a given lacunary sequence $\theta=\left\{k_{r}\right\}$, a sequence $x=\left\{x_{k}\right\}$ is lacunary statistical bounded if and only if there exists a bounded sequence $y=\left\{y_{k}\right\}$ such that $x_{k}=y_{k}$ a.a.k w.r.t. $\theta$. 
Proof First suppose that $x=\left\{x_{k}\right\}$ is a lacunary statistical bounded sequence. Then there exists $M \geq 0$ such that $\delta^{(\theta)}(K)=0$ where $K=\left\{k \in \mathbb{N}:\left|x_{k}\right|>M\right\}$. Let

$$
y_{k}= \begin{cases}x_{k}, & \text { if } k \notin K \\ 0, & \text { otherwise }\end{cases}
$$

Then $y=\left\{y_{k}\right\} \in \ell_{\infty}$ and $y_{k}=x_{k}$ a.a.k w.r.t. $\theta$.

Conversely, as $y=\left\{y_{k}\right\} \in \ell_{\infty}$ so there exists $L \geq 0$ such that $\left|y_{k}\right| \leq L$ for all $k \in \mathbb{N}$. Let $D=\left\{k \in \mathbb{N}: x_{k} \neq y_{k}\right\}$. As $\delta^{(\theta)}(D)=0$, so $\left|x_{k}\right| \leq L$ a.a.k w.r.t. $\theta$.

Corollary 2.8 Every lacunary statistical bounded sequence has a bounded subsequence.

A decomposition theorem for statistical boundedness was given by Tripathy [32]. We now give a lacunary analog of this result.

Theorem 2.9 (Decomposition theorem) If $x=\left\{x_{k}\right\}$ is a lacunary statistical bounded sequence, then there exists a bounded sequence $y=\left\{y_{k}\right\}$ and a lacunary statistical null sequence $z=\left\{z_{k}\right\}$ such that $x=y+z$. However, this decomposition is not unique.

Proof As $x=\left\{x_{k}\right\}$ is a lacunary statistical bounded sequence, so there exists $M>0$ such that $\delta^{\theta}(A)=0$, where $A=\left\{k \in \mathbb{N}:\left|x_{k}\right|>M\right\}$. Define sequences $y=\left\{y_{k}\right\}$ and $z=\left\{z_{k}\right\}$ as follows:

$$
\begin{aligned}
& y_{k}= \begin{cases}x_{k}, & \text { if } k \in \mathbb{N}-A ; \\
0, & \text { if } k \in A,\end{cases} \\
& z_{k}= \begin{cases}0, & \text { if } k \in \mathbb{N}-A ; \\
x_{k}, & \text { if } k \in A .\end{cases}
\end{aligned}
$$

Clearly $x=y+z$ where $y$ is a bounded sequence and $z$ is a lacunary statistical null sequence, i.e., $S_{\theta}(b) \subset \ell_{\infty}+c_{\theta}^{0}$ where $c_{\theta}^{0}$ denotes the set of all lacunary statistical null sequences. As $\ell_{\infty}, c_{\theta}^{0} \subset S_{\theta}(b)$, so $\ell_{\infty}+c_{\theta}^{0} \subset S_{\theta}(b)$. Consequently, we have $S_{\theta}(b)=\ell_{\infty}+c_{\theta}^{0}$. Using the fact that $\phi \subset \ell_{\infty} \cap c_{\theta}^{0}$, where $\phi$ is the space of finitely non-zero scalar sequences, we have $S_{\theta}(b) \neq \ell_{\infty} \oplus c_{\theta}^{0}$, i.e., the decomposition is not unique.

\section{Theorem 2.10}

(a) $S_{\theta}(b)$ is normal and hence monotone.

(b) $S_{\theta}(b)$ is a sequence algebra.

The proof is easy and so omitted.

Proposition 2.11 $\left[S_{\theta}(b)\right]^{\alpha}=\left[S_{\theta}(b)\right]^{\beta}=\phi$, the space of finitely non-zero scalar sequences.

Proof To show that $\left[S_{\theta}(b)\right]^{\alpha}=\phi$, it is sufficient to show that $\left[S_{\theta}(b)\right]^{\alpha} \subset \phi$ since $\phi \subset\left[S_{\theta}(b)\right]^{\alpha}$ obviously. Let $\left\{a_{k}\right\} \in\left[S_{\theta}(b)\right]^{\alpha}$. Then $\sum_{k}\left|a_{k} x_{k}\right|<\infty$ for all $x=\left\{x_{k}\right\} \in S_{\theta}(b)$. Suppose $\left\{a_{k}\right\} \notin$ $\phi$, i.e., $\left\{a_{k}\right\}$ has infinitely many non-zero terms. Following Lemma 5 of [12], for each $r \in \mathbb{N}$, if $I_{r}$ contains a $k$ such that $a_{k} \neq 0$, let $m(r)$ be the least such $k$; otherwise leave $m(r)$ undefined. 
Thus there are infinitely many $m(r)$ 's and $m(r) \in I_{r}$. Now define $x_{k}=\frac{1}{\left|a_{k}\right|}$ if $k=m(r)$ for some $r=1,2,3, \ldots$ and $x_{k}=0$ otherwise. Now $\frac{1}{h_{r}}\left|\left\{k \in I(r):\left|x_{k}\right|>0\right\}\right|=\frac{1}{h_{r}} \mid\left\{k \in I(r):\left|x_{k}\right| \neq\right.$ $0\} \mid=\frac{1}{h_{r}} \rightarrow 0$ as $r \rightarrow \infty$ and so $\left\{x_{k}\right\} \in S_{\theta}(b)$. But $\sum_{I_{r}}\left|a_{k} x_{k}\right|=1$ for infinitely many $r$ and so $\sum_{k}\left|a_{k} x_{k}\right|=\infty$.

In view of the fact [35, p.52] that for a monotone sequence space, $\alpha$ - and $\beta$-dual spaces coincide, we have $\left[S_{\theta}(b)\right]^{\beta}=\left[S_{\theta}(b)\right]^{\alpha}=\phi$.

Corollary 2.12 $S_{\theta}(b)$ is not perfect.

Proof As $\left[S_{\theta}(b)\right]^{\alpha \alpha}=\phi^{\alpha}=\omega$, so $S_{\theta}(b)$ is not a perfect space.

\section{Lacunary statistical boundedness versus statistical boundedness}

In this section, we study the inclusions $S_{\theta}(b) \subset S(b)$ and $S(b) \subset S_{\theta}(b)$ under certain restrictions on $\theta=\left\{k_{r}\right\}$ and characterize those $\theta$ for which $S_{\theta}(b)=S(b)$.

Lemma 3.1 For any lacunary sequence $\theta, S(b) \subset S_{\theta}(b)$ if and only if $\liminf _{r} q_{r}>1$.

Proof (Sufficiency). If $\liminf _{r} q_{r}>1$, then there exists $\delta>0$ such that $q_{r} \geq 1+\delta$ for sufficiently large $r$. Since $h_{r}=k_{r}-k_{r-1}$, so we have $\frac{h_{r}}{k_{r}} \geq \frac{\delta}{1+\delta}$ and $\frac{k_{r-1}}{h_{r}} \leq \frac{1}{\delta}$. For $\left\{x_{k}\right\} \in S_{\theta}(b)$, there exists $M>0$ such that $\lim _{r \rightarrow \infty} \frac{1}{h_{r}}\left|\left\{k \in I_{r}:\left|x_{k}\right|>M\right\}\right|=0$.

Now for sufficiently large $r$, we have

$$
\begin{aligned}
\frac{1}{k_{r}}\left|\left\{k \leq k_{r}:\left|x_{k}\right|>M\right\}\right| & \geq \frac{h_{r}}{k_{r}} \frac{1}{h_{r}}\left|\left\{k \in I_{r}:\left|x_{k}\right|>M\right\}\right| \\
& \geq \frac{\delta}{1+\delta} \frac{1}{h_{r}}\left|\left\{k \in I_{r}:\left|x_{k}\right|>M\right\}\right| .
\end{aligned}
$$

This proves the sufficiency.

(Necessity). Assume that $\liminf _{r} q_{r}=1$. Proceeding as in Lemma 2.1 of [28], we can select a subsequence $\left\{k_{r(j)}\right\}$ of $\theta=\left\{k_{r}\right\}$ satisfying

$$
\frac{k_{r(j)}}{k_{r(j)-1}}<1+\frac{1}{j} \quad \text { and } \quad \frac{k_{r(j)-1}}{k_{r(j-1)}}>j, \quad \text { where } r(j) \geq r(j-1)+2 .
$$

Define $x=\left\{x_{k}\right\}$ by

$$
x_{i}= \begin{cases}i, & \text { if } i \in I_{r(j)}, \text { for some } j=1,2,3, \ldots ; \\ 0, & \text { otherwise }\end{cases}
$$

Now for any $M>0$, there exists $j_{0} \in \mathbb{N}$ (actually there are infinitely many $j_{0}$ ) such that $k_{r\left(j_{0}\right)-1}>M$ and we have

$$
\frac{1}{h_{r\left(j_{0}\right)}}\left|\left\{k \in I_{r\left(j_{0}\right)}:\left|x_{k}\right|>M\right\}\right| \geq \frac{1}{h_{r\left(j_{0}\right)}}\left|\left\{k \in I_{r\left(j_{0}\right)}:\left|x_{k}\right|>k_{r\left(j_{0}\right)-1}\right\}\right|
$$


i.e., $\frac{1}{h_{r(j)}}\left|\left\{k \in I_{r(j)}:\left|x_{k}\right|>M\right\}\right|=1$ for all $j \geq j_{0}$. Also, for $r \neq r(j), \frac{1}{h_{r}}\left|\left\{k \in I_{r}:\left|x_{k}\right|>M\right\}\right|=0$. Thus $x=\left\{x_{k}\right\} \notin S_{\theta}(b)$.

Now for any $t$ sufficiently large integer, we can find the unique $j$ for which $k_{r(j)-1}<t \leq$ $k_{r(j+1)-1}$ and write

$$
\frac{1}{t}\left|\left\{k \leq t:\left|x_{k}\right|>\frac{1}{2}\right\}\right| \leq \frac{k_{r(j-1)}+h_{r(j)}}{k_{r(j)-1}}<\frac{1}{j}+\frac{1}{j}=\frac{2}{j} .
$$

Since $t \rightarrow \infty$ implies $j \rightarrow \infty$, we have $\left\{x_{k}\right\} \in S(b)$.

Remark 3.2 The sequence $\left\{x_{k}\right\}$, constructed in the necessity part of above lemma, is an example of a statistical bounded sequence which is not lacunary statistical bounded.

Lemma 3.3 For any lacunary sequence $\theta, S_{\theta}(b) \subset S(b)$ if and only if $\lim \sup _{r} q_{r}<\infty$.

Proof The proof of sufficiency part can be established following a similar technique to Lemma 3 of [29]. For necessity, we assume that $\limsup _{r} q_{r}=\infty$. Following Lemma 2.2 of [28], we can select a subsequence $\left\{k_{r(j)}\right\}$ of the lacunary sequence $\theta$ such that $q_{r(j)}>j$.

Define $x=\left\{x_{k}\right\}$ as

$$
x_{i}= \begin{cases}i, & \text { if } k_{r(j)-1}<i \leq 2 k_{r(j)-1}, \text { for some } j=1,2, \ldots \\ 0, & \text { otherwise }\end{cases}
$$

Then $\tau_{r(j)}=\frac{1}{h_{r(j)}}\left|\left\{i \in I_{r(j)}:\left|x_{i}\right|>\frac{1}{2}\right\}\right|=\frac{k_{r(j)-1}}{k_{r(j)}-k_{r(j)-1}}<\frac{1}{j-1}$ and, if $r \neq r_{j}, \tau_{r}=0$. Hence $\left\{x_{k}\right\} \in S_{\theta}(b)$. However, for any real $M>0$, there exists some $j_{0}$ such that $k_{r(j)-1}>M$ for all $j \geq j_{0}$;

$$
\begin{aligned}
\frac{1}{2 k_{r(j)-1}}\left|\left\{i \leq 2 k_{r(j)-1}:\left|x_{i}\right|>M\right\}\right| & \geq \frac{1}{2 k_{r(j)-1}}\left|\left\{i \leq 2 k_{r(j)-1}:\left|x_{i}\right|>k_{r(j)-1}\right\}\right| \\
& \geq \frac{1}{2}
\end{aligned}
$$

and this is true for all $j \geq j_{0}$. Thus $\left\{x_{k}\right\} \notin S(b)$.

Remark 3.4 The sequence $\left\{x_{k}\right\}$, constructed in the necessity part of above lemma, is an example of a lacunary statistical bounded sequence which is not statistical bounded.

Combining Lemma 3.1 and Lemma 3.3 we have the following.

Theorem 3.5 Let $\theta$ be a lacunary sequence. Then $S_{\theta}(b)=S(b)$ if and only if $1<\liminf _{r} q_{r} \leq$ $\lim \sup _{r} q_{r}<\infty$.

\section{Theorem 3.6}

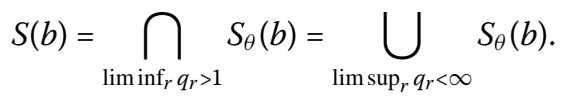

Proof In view of Lemma 3.1, we have $S(b) \subset \bigcap_{\liminf _{r} q_{r}>1} S_{\theta}(b)$. Suppose, if possible, $x=$ $\left\{x_{k}\right\} \in \bigcap_{\liminf _{r} q_{r}>1} S_{\theta}(b)$ but $x \notin S(b)$. We have $\left\{x_{k}\right\} \in S_{\theta}(b)$ for all $\theta=\left\{k_{r}\right\}$ with $\liminf _{r} q_{r}>1$. 
If we take $\theta=\left\{2^{r}\right\}$, then in view of Theorem 3.5 we have $S_{\theta}(b)=S(b)$ and so $x \notin S(b)$, contrary to our supposition. Hence $S(b)=\bigcap_{\liminf _{r} q_{r}>1} S_{\theta}(b)$. The remaining part can be proved similarly and hence is omitted.

Remark 3.7 It is well known that every subsequence of a bounded sequence is bounded. However, for lacunary statistical bounded sequences this is no longer true. This can be verified by the following example.

Example 3.8 Let $\theta=\left\{k_{r}\right\}$ be a lacunary sequence with $\liminf _{r} q_{r}>1$ and consider the sequence $x=\left\{x_{k}\right\}$ as

$$
x_{k}= \begin{cases}k, & \text { if } k \text { is a square; } \\ 0, & \text { if } k \text { is not a square }\end{cases}
$$

i.e., $x=\left\{x_{k}\right\}=(1,0,0,4,0,0,0,0,9, \ldots)$. Then $\left\{x_{k}\right\} \in S(b) \subset S_{\theta}(b)$. However, $\left\{y_{k}\right\}=(1,4,9$, ...) is a subsequence of the lacunary statistical bounded sequence $\left\{x_{k}\right\}$ but is not lacunary statistical bounded.

We now characterize those subsequences of a lacunary statistical bounded sequence which are themselves lacunary statistical bounded. Before doing so, we introduce the following definition.

Definition 3.9 Let $\theta$ be a lacunary sequence. A subset $A$ of $\mathbb{N}$ is said to be lacunary statistical dense or $\theta$ dense if $\delta^{\theta}(A)=1$. A subsequence of a sequence is said to be lacunary statistical dense if the set of all indices of its elements is lacunary statistical dense.

In view of Theorem 2.1 of Burgin and Duman [27] we state the following result without proof.

Theorem 3.10 A sequence is lacunary statistical bounded if and only if every lacunary statistical dense subsequence of it is lacunary statistical bounded.

\section{Inclusion between two lacunary methods of statistical boundedness}

Our first result shows that if $\beta$ is a refinement of $\theta$, then $S_{\beta}(b) \subset S_{\theta}(b)$.

We state the following result, which can be established following the technique of Theorem 7 of Fridy and Orhan [29].

Theorem 4.1 If $\beta$ is a lacunary refinement of $\theta$ and $\left\{x_{k}\right\} \in S_{\beta}(b)$, then $\left\{x_{k}\right\} \in S_{\theta}(b)$, i.e., $S_{\beta}(b) \subset S_{\theta}(b)$.

Following Li [13], we state the next result without proof in which we impose certain restriction on a refinement $\beta$ of $\theta$ so as to have the reverse inclusion i.e., $S_{\theta}(b) \subset S_{\beta}(b)$.

Theorem 4.2 Suppose $\beta=\left\{\ell_{r}\right\}$ is a lacunary refinement of the lacunary sequence $\theta=\left\{k_{r}\right\}$. Let $I_{r}=\left(k_{r-1}, k_{r}\right]$ and $J_{r}=\left(\ell_{r-1}, \ell_{r}\right], r=1,2,3, \ldots$. If there exists $\delta>0$ such that $\frac{\left|J_{j}\right|}{\left|I_{i}\right|} \geq \delta$ for every $J_{j} \subset I_{i}$, then $S_{\theta}(b) \subset S_{\beta}(b)$.

The following result is a consequence of Theorem 4.1 and Theorem 4.2. 
Corollary 4.3 Under the hypothesis of Theorem 4.2, we have $S_{\theta}(b)=S_{\beta}(b)$.

The next theorem provides a sufficient condition for lacunary sequences $\beta=\left\{\ell_{r}\right\}$ and $\theta=\left\{k_{r}\right\}$ to yield the inclusion relation $S_{\theta}(b) \subset S_{\beta}(b)$.

In view of Theorem 2 of $\mathrm{Li}$ [13] we state the following result without proof.

Theorem 4.4 Suppose $\beta=\left\{\ell_{r}\right\}, \theta=\left\{k_{r}\right\}$ are two lacunary sequences. Let $I_{r}=\left(k_{r-1}, k_{r}\right]$, $J_{r}=\left(\ell_{r-1}, \ell_{r}\right], r=1,2,3, \ldots$, and $I_{i j}=I_{i} \cap J_{j}, i, j=1,2,3, \ldots$. If there exists $\delta>0$ such that $\frac{\left|I_{i j}\right|}{\left|I_{i}\right|} \geq \delta$ for every $i, j=1,2,3, \ldots$, provided $I_{i j} \neq \phi$, then $S_{\theta}(b) \subset S_{\beta}(b)$.

Remark 4.5 If the condition in Theorem 4.4 is replaced by $\frac{\left|I_{i j}\right|}{\left|J_{j}\right|} \geq \delta$ for every $i, j=1,2,3, \ldots$, provided $I_{i j} \neq \phi$, then it can be seen that $S_{\beta}(b) \subset S_{\theta}(b)$.

Combining Remark 4.5 and Theorem 4.4, we get the following.

Theorem 4.6 Suppose $\beta=\left\{\ell_{r}\right\}, \theta=\left\{k_{r}\right\}$ are two lacunary sequences. Let $I_{r}=\left(k_{r-1}, k_{r}\right]$, $J_{r}=\left(\ell_{r-1}, \ell_{r}\right], r=1,2,3, \ldots$, and $I_{i j}=I_{i} \cap J_{j}, i, j=1,2,3, \ldots$. If there exists $\delta>0$ such that $\frac{\left|I_{i j}\right|}{\left|I_{i}\right|+\left|I_{j}\right|} \geq \delta$ for every $i, j=1,2,3, \ldots$, provided $I_{i j} \neq \phi$, then $S_{\theta}(b)=S_{\beta}(b)$.

Competing interests

The authors declare that there is no conflict of interests regarding the publication of this paper.

Authors' contributions

All authors contributed equally to the writing of this paper. All authors read and approved the final manuscript.

\section{Author details}

${ }^{1}$ Department of Mathematics, Kurukshetra University, Kurukshetra, 136119, India. ${ }^{2}$ Department of Mathematics, Arya P. G. College, Panipat, 132103, India. ${ }^{3}$ Department of Mathematics, Faculty of Science, King Abdulaziz University, P.O. Box 80203, Jeddah, 21589, Saudi Arbia. ${ }^{4}$ Department of Mathematics, Universiti Putra Malaysia, Serdang, Selangor 43400, Malaysia. ${ }^{5}$ Institute for Mathematical Research, Universiti Putra Malaysia, Serdang, Selangor 43400, Malaysia.

\section{Acknowledgements}

The authors are grateful to the referee for his/her valuable comments and suggestions, which have improved the presentation of the paper

Received: 27 February 2014 Accepted: 30 July 2014 Published: 21 Aug 2014

\section{References}

1. Zygmund, A: Trigonometric Series, 2nd edn. Cambridge University Press, Cambridge (1979)

2. Fast, H: Sur la convergence statistique. Colloq. Math. 2, 241-244 (1951)

3. Schoenberg, IJ: The integrability of certain functions and related summability methods. Am. Math. Mon. 66, 361-375 (1959)

4. Buck, RC: Generalized asymptotic density. Am. J. Math. 75, 335-346 (1953)

5. Bhardwaj, VK, Bala, I: On weak statistical convergence. Int. J. Math. Math. Sci. 2007, Article ID 38530 (2007)

6. Bhardwaj, VK, Bala, I: On lacunary generalized difference sequence spaces defined by Orlicz functions in a seminormed space and $\Delta_{a}^{m}$-lacunary statistical convergence. Demonstr. Math. 41(2), 415-424 (2008)

7. Çinar, M, Karakaş, M, Et, M: On pointwise and uniform statistical convergence of order $\alpha$ for sequences of functions. Fixed Point Theory Appl. 2013, 33 (2013)

8. Demirci, K: A criterion for A statistical convergence. Indian J. Pure Appl. Math. 29(5), 559-564 (1998)

9. Et, M, Çinar, M, Karakaş, M: On $\lambda$-statistical convergence of order $\alpha$ of sequences of function. J. Inequal. Appl. 2013 $204(2013)$

10. Fridy, JA: On statistical convergence. Analysis 5, 301-313 (1985)

11. Fridy, JA: Statistical limit points. Proc. Am. Math. Soc. 118, 1187-1192 (1993)

12. Fridy, JA, Orhan, C: Lacunary statistical summability. J. Math. Anal. Appl. 173, 497-504 (1993)

13. Li, J: Lacunary statistical convergence and inclusion properties between lacunary methods. Int. J. Math. Math. Sci. 23(3), 175-180 (2000)

14. Mohiuddine, SA, Aiyub, M: Lacunary statistical convergence in random 2-normed spaces. Appl. Math. Inf. Sci. 6(3), 581-585 (2012)

15. Mohiuddine, SA, Alghamdi, MA: Statistical summability through a lacunary sequence in locally solid Riesz spaces. J. Inequal. Appl. 2012, 225 (2012) 
16. Mohiuddine, SA, Alotaibi, A, Mursaleen, M: A new variant of statistical convergence. J. Inequal. Appl. 2013, 309 (2013)

17. Mohiuddine, SA, Alotaibi, A, Mursaleen, M: Statistical convergence through de la Vallee-Poussin mean in locally solid Riesz spaces. Adv. Differ. Equ. 2013, 66 (2013)

18. Mohiuddine, SA, Hazarika, B, Alotaibi, A: Double lacunary density and some inclusion results in locally solid Riesz spaces. Abstr. Appl. Anal. 2013, Article ID 507962 (2013)

19. Šalàt, T: On statistically convergent sequences of real numbers. Math. Slovaca 30(2), 139-150 (1980)

20. Savaş, E, Mohiuddine, SA: $\bar{\lambda}$-statistically convergent double sequences in probabilistic normed spaces. Math. Slovaca 62(1), 99-108 (2012)

21. Tripathy, BC, Baruah, A: Lacunary statistically convergent and lacunary strongly convergent generalized difference sequences of fuzzy real numbers. Kyungpook Math. J. 50(4), 565-574 (2010)

22. Tripathy, BC, Dutta, H: On some lacunary difference sequence spaces defined by a sequence of Orlicz functions and $q$-lacunary $\Delta_{m}^{n}$-statistical convergence. An. Univ. 'Ovidius' Constanţa, Ser. Mat. 20(1), 417-430 (2012)

23. Tripathy, BC, Dutta, AJ: Lacunary bounded variation sequence of fuzzy real numbers. J. Intell. Fuzzy Syst. 24(1), 185-189 (2013)

24. Tripathy, BC, Hazarika, B, Choudhary, B: Lacunary /-convergent sequences. Kyungpook Math. J. 52(4), 473-482 (2012)

25. Tripathy, BC, Mahanta, S: On a class of generalized lacunary difference sequence spaces defined by Orlicz function. Acta Math. Appl. Sinica (Engl. Ser.) 20(2), 231-238 (2004)

26. Niven, I, Zuckerman, HS: An Introduction to the Theory of Numbers, 4th edn. Wiley, New York (1980)

27. Burgin, M, Duman, O: Statistical convergence and convergence in statistics. arXiv:math/0612179 [math.GM]

28. Freedman, AR, Sember, JJ, Raphael, M: Some Cesàro type summability spaces. Proc. Lond. Math. Soc. 37, 508-520 (1978)

29. Fridy, JA, Orhan, C: Lacunary statistical convergence. Pac. J. Math. 160, 43-51 (1993)

30. Freedman, AR, Sember, JJ: Densities and summability. Pac. J. Math. 95, 293-305 (1981)

31. Fridy, JA, Orhan, C: Statistical limit superior and limit inferior. Proc. Am. Math. Soc. 125(12), 3625-3631 (1997)

32. Tripathy, BC: On statistically convergent and statistically bounded sequences. Bull. Malays. Math. Soc. 20, 31-33 (1997)

33. Bhardwaj, VK, Gupta, S: On some generalizations of statistical boundedness. J. Inequal. Appl. 2014, 12 (2014)

34. Cooke, RG: Infinite Matrices and Sequence Spaces. Macmillan, London (1950)

35. Kamthan, PK, Gupta, M: Sequence Spaces and Series. Dekker, New York (1981)

36. Köthe, G, Toeplitz, O: Lineare Räume mit unendlich vielen Koordinaten und Ringe unendlicher Matrizen. J. Reine Angew. Math. 171, 193-226 (1934)

37. Chillingworth, HR: Generalized 'dual' sequence spaces. Ned. Akad. Wet. Indag. Math. 20, 307-315 (1958)

10.1186/1029-242X-2014-311

Cite this article as: Bhardwaj et al.: On lacunary statistical boundedness. Journal of Inequalities and Applications

2014, 2014:311

\section{Submit your manuscript to a SpringerOpen ${ }^{\circ}$ journal and benefit from:}

- Convenient online submission

- Rigorous peer review

Immediate publication on acceptance

- Open access: articles freely available online

- High visibility within the field

- Retaining the copyright to your article 